\title{
Disfagia e melhoria do estado nutricional: Características tecno-funcionais de
}

\section{espessantes comerciais}

Dysphagia and improvement of nutritional status: Techno-functional characteristics of commercial

\author{
thickeners
}

Disfagia y mejora del estado nutricional: Características tecno-funcionales de los espesantes comerciales

Cintia Mitie Ikeda

ORCID: https://orcid.org/0000-0002-5973-1663 Universidade Federal de Alfenas, Brasil E-mail: cinthya_ikeda@hotmail.com

Bruna Lago Tagliapietra ORCID: https://orcid.org/0000-0003-3041-4768 Universidade Estadual de Campinas, Brasil E-mail: bruna_tagliapietra@hotmail.com

Pedro Henrique Campelo ORCID: https://orcid.org/0000-0002-4048-3858 Universidade Federal do Amazonas, Brasil E-mail: pedrocampelo@ufam.edu.br

Maria Teresa Pedrosa Silva Clerici ORCID: https://orcid.org/0000-0002-8445-336X Universidade Estadual de Campinas, Brasil E-mail: mclerici@unicamp.br

Luciano Bruno de Carvalho Silva ORCID: https://orcid.org/0000-0002-8056-4396 Universidade Federal de Alfenas, Brasil E-mail: luciano@unifal-mg.edu.br

\begin{abstract}
Resumo
A disfagia se caracteriza por uma disfunção no processo de deglutição, que prejudica a alimentação oral autônoma e segura. Para minimizar os efeitos negativos alimentos de textura modificada e bebidas espessadas são utilizadas. Com isso, esse estudo objetivou comparar as viscosidades de espessantes alimentares comerciais quando dissolvidos em preparações líquidas. Três espessantes a base de amido foram utilizados para espessar cinco preparações (água, suco de laranja, leite integral, leite espessado e café). Foram avaliados a informação nutricional, microscopia, $\mathrm{pH}$, poder de inchamento e solubilidade dos grânulos e a viscosidade das preparações em função do tempo e da temperatura. Todos os espessantes apresentaram alta porcentagem de carboidrato, sódio e presença de amido modificado. Os espessantes promoveram mudanças no $\mathrm{pH}$ das preparações e a presença de amido de milho modificado mostrou aumento da solubilidade e poder de inchamento. Em relação às variações de temperatura, valores mais baixos aumentaram a viscosidade, e a viscosidade aumentou com o repouso, provavelmente pela hidratação contínua do agente espessante. Quanto maior a consistência que se deseja atingir, maiores quantidades de produto precisam ser adicionadas. As preparações alimentares espessadas apresentaram viscosidades diferentes, dependendo da matriz alimentar, temperatura, tempo e da quantidade de produto adicionado.
\end{abstract}

Palavras-chave: Deglutição; Espessante alimentar; Viscosidade; Gomas; Amido.

\begin{abstract}
Dysphagia is characterized by a dysfunction in the swallowing process, which impairs safe and autonomous oral feeding. To minimize the negative effects, modified texture foods and thickened drinks are used. This study aimed to compare the viscosities of commercial food thickeners when dissolved in liquid preparations. Three starch-based thickeners were used to thicken five preparations (water, orange juice, milk, thickened milk, and coffee). Nutritional information, microscopy, $\mathrm{pH}$, swelling power, and solubility of the granules and the viscosity of the preparations as a function of time and temperature were evaluated. All thickeners had a high percentage of carbohydrates, sodium, and the presence of modified starch. The thickeners promoted changes in the $\mathrm{pH}$ of the preparations and the presence of modified corn starch showed an increase in solubility and swelling power. About temperature variations, lower values increased viscosity, and viscosity increased with rest, probably due to the continuous hydration of the thickening agent. The greater the consistency you want to achieve, the greater quantities of product need to be added. The
\end{abstract}


thickened food preparations showed different viscosities, depending on the food matrix, temperature, time and the amount of product added.

Keywords: Deglutition; Food thickener; Viscosity; Gums; Starch.

\begin{abstract}
Resumen
La disfagia se caracteriza por una disfunción en el proceso de deglución, que perjudica la alimentación oral segura y autónoma. Para minimizar los efectos negativos, se utilizan alimentos con textura modificada y bebidas espesas. En este sentido, este estudio tuvo como objetivo comparar las viscosidades de espesantes de alimentos comerciales al ser disueltos en preparaciones líquidas. Se utilizaron tres espesantes a base de almidón para espesar cinco preparaciones (agua, jugo de naranja, leche entera, leche espesa y café). Donde se evaluaron la información nutricional, la microscopía, el $\mathrm{pH}$, poder de hinchamiento y la solubilidad de los gránulos, así como la viscosidad de las preparaciones en función del tiempo y la temperatura. Todos los espesantes mostraron un alto porcentaje de carbohidratos, sodio y la presencia de almidón modificado. Los espesantes promovieron cambios en el $\mathrm{pH}$ de las preparaciones, y la presencia de almidón de maíz modificado produjo un aumento en la solubilidad y el poder de hinchamiento. En cuanto a las variaciones de temperatura, los valores más bajos aumentaron la viscosidad, la cual se vio incrementada con el reposo, probablemente debido a la hidratación continua del agente espesante. Cuanto mayor sea la consistencia que se desee lograr, mayores cantidades del producto deberán ser agregadas. Los preparados alimenticios espesados mostraron diferentes viscosidades, dependiendo de la matriz del alimento, la temperatura, el tiempo y la cantidad de producto agregado.
\end{abstract}

Palabras clave: Deglución; Espesante de alimentos; Viscosidad; Gomas; Almidón.

\title{
1. Introdução
}

A deglutição é um processo complexo que envolve estruturas relacionadas com a cavidade oral, faringe, laringe e do esôfago, submetidos a um controle neuromuscular que permite o transporte do bolo alimentar da boca até o estômago, sem permitir a entrada de qualquer substância para a via aérea (Ertekin \& Aydogdu, 2003). A dificuldade ou incapacidade de mastigar ou engolir alimentos ou líquidos devido ao enfraquecimento dos músculos usados para engolir é chamada de disfagia (Sukkar et al., 2018).

Pessoas com disfagia têm dificuldade de controlar o fluxo de líquidos durante a passagem pela faringe, resultando em comprometimento da proteção das vias aéreas (Cichero, 2013). Além disso, prejudica a alimentação oral autônoma e segura, podendo desencadear várias consequências negativas a saúde, como: ingestão oral reduzida (resultando em desnutrição), desidratação, imunodeficiência, pneumonia aspirativa, penetração laríngea e asfixia (Andersen et al., 2013). Esse distúrbio da deglutição pode ser causado por anormalidades anatômicas ou fisiológicas que podem ter uma base neurológica ou estrutural, como acidente vascular cerebral, doença do neurônio motor, doença de Parkinson, câncer de cabeça e pescoço e outros (Logemann, 2007).

Mais recente, estudos indicam que a disfagia tem se feito presente em pacientes infectados pelo COVID-19 (síndrome respiratória grave causada pelo vírus SARS-CoV-2) (Ellul et al., 2020; Phua et al., 2020; Lima et al., 2020). Pacientes com COVID-19 podem apresentar dificuldades de alimentação devido a diversos fatores, como aumento do esforço respiratório, uso de dispositivos de suporte ventilatório, comprometimento neurológico e disfagia pós-extubação (Fernández et al., 2020). Estudo realizado no Brasil, por Lima et al. (2020) com pacientes internados em UTI, submetidos à intubação orotraqueal prolongada ( $\geq 48 \mathrm{~h}$ ), demonstra que quase $20 \%$ dos pacientes não apresentam condições mínimas de alimentação segura, necessitando de forma alternativa de alimentação e que $83 \%$ dos pacientes necessitam de até três intervenções fonoaudiólogas para a recuperação dos padrões seguros de deglutição.

A nutrição é um aspecto importante do cuidado ao paciente com disfagia, e o suporte nutricional adequado tem o potencial de reverter ou mitigar as consequências adversas do mau estado nutricional (Wei et al., 2021). Em função dos efeitos negativos, diversas intervenções são utilizadas no manejo da disfagia, incluindo modificações na textura dos alimentos e líquidos, como estratégias para tornar o processo de deglutição mais lento e, assim, mais seguro e eficiente (Dobak \& Kelly, 2020). 
Vários agentes espessantes são utilizados para melhorar a textura e a consistência de determinados alimentos, como amidos, gomas, pectinas, derivados de celulose entre outros (Moret-Tatay et al., 2015). Os espessantes alimentares comerciais estão disponíveis na forma de pós, que podem ser adicionados a diversas preparações. No entanto, alguns estudos demonstram que os espessantes alimentares comerciais utilizados no tratamento da disfagia apresentam propriedades e poder espessante distintos, quando misturados em diferentes matrizes alimentares (Adeleye \& Rachal, 2007; Sopade et al., 2008; Moret-Tatay et al., 2015).

O controle da viscosidade dos alimentos espessados é importante para o manejo e tratamento dos pacientes disfágicos, visto que melhora o controle oral sobre o bolo alimentar e possibilita maior tempo para que o reflexo da deglutição seja desencadeado (Kim \& Yoo, 2018). Contudo, a falta de padrões claros para as dietas modificadas e para líquidos espessados levou a publicação do guia "National Disphagia Diet" (NDD) (National Dysphagia Diet Task Force, 2002). O NDD incluiu recomendações para rotulagem e taxas de viscosidade para cada um dos quatro níveis de consistências recomendados no tratamento da disfagia, sendo categorizados como: "Finos", com viscosidade de 1 a $50 \mathrm{cP}$; "Néctar" de 51 a $350 \mathrm{cP}$; "Mel" de 351 a 1750 cP, e "Pudim" superior a 1750 cP. Os líquidos finos dificultam a deglutição de pacientes que apresentam controle laríngeo reduzido e os líquidos espessados produzem consistência mais segura (Schmidt \& Oliveira, 2015).

Atualmente, há disponível uma ampla variedade de espessantes alimentares comerciais, porém há poucas informações sobre as diferenças na viscosidade dos produtos, que pode estar relacionada com a composição química dos mesmos, o tipo de líquido e a matriz alimentar a ser espessada, o que dificulta atingir a viscosidade ideal para os disfágicos. Assim, o presente estudo visa comparar as viscosidades de três espessantes alimentares comerciais quando dissolvidos em cinco preparações líquidas: água, suco de laranja, leite integral, leite espessado e café.

\section{Metodologia}

\subsection{Caracterização das preparações líquidas e dos espessantes alimentares comerciais}

Trata-se de uma pesquisa quantitativa (Pereira et al., 2018), onde foram estudados três espessantes alimentares comerciais à base de amido, utilizados no tratamento de disfagia: Resource Thicken Up®, da empresa Novartis (amido modificado, não contém glúten), Nutilis®, da empresa Support (amido modificado, espessantes gomas tara, xantana e guar, não contém glúten) e Thick \& Easy®, da empresa Fresenius (amido modificado e maltodextrina).

As preparações líquidas escolhidas para serem espessadas foram: água, suco de laranja, leite integral, leite espessado (100 mL de leite integral com $30 \mathrm{~g}$ de Sustagem ${ }^{\circledR}$ ) e café. Foi realizada a caracterização nutricional das preparações líquidas e dos espessantes alimentares comerciais por meio da informação nutricional disponibilizada no rótulo pelos fabricantes.

\subsection{Microscopia}

A microscopia foi realizada em microscópio óptico, da marca Zeiss, em aumento de 40 vezes. As amostras foram coradas com solução de lugol, para melhor visualização do amido modificado, uma vez que todas as amostras apresentavam este ingrediente.

\section{$2.3 \mathrm{pH}$}

Para a determinação do $\mathrm{pH}$ foram pesados $5 \mathrm{~g}$ da amostra e dispersada em $100 \mathrm{~mL}$ de água destilada previamente fervida e resfriada ou $100 \mathrm{~mL}$ das respectivas preparações líquidas: café, leite, leite espessado e suco de laranja. Agitou-se a suspensão por 30 minutos com intervalos de repouso de 5 minutos. $\mathrm{O}$ pH foi medido em potenciômetro calibrado a $20^{\circ} \mathrm{C}$ imediatamente após a agitação. 


\subsection{Poder de inchamento e solubilidade dos grânulos}

A análise de solubilidade dos grânulos foi realizada conforme Silva et al., (2006) com adaptações. As amostras a $25^{\circ} \mathrm{C}$ foram colocadas em tubos de centrífugas de $15 \mathrm{~mL}$, previamente pesados, contendo uma suspensão em $10 \mathrm{~mL}$ de água deionizada e $62,5 \mathrm{mg}$ de amostra. Após 30 minutos de agitação, a suspensão foi centrifugada a $2.200 \mathrm{rpm}$ por 10 minutos para a separação de fases. $\mathrm{O}$ sobrenadante foi coletado e seco em estufa a $105^{\circ} \mathrm{C}$ até peso constante, para quantificação da fração solúvel. Os tubos contendo os grânulos de amido intumescido foram pesados para a determinação do poder de inchamento, e o resultado expresso em vezes de ganho de peso em relação a massa de amostra inicial.

\subsection{Análise das viscosidades}

Para a uniformidade e reprodutibilidade das preparações líquidas espessadas foi realizado a padronização das medidas caseiras, na qual uma colher de chá equivale a 5 gramas. As medidas caseiras padronizadas utilizadas para as preparações neste estudo foram: 1, 2, 3 e 4 colheres de chá de cada espessante alimentar comercial.

As viscosidades aparentes $(\eta)$ foram determinadas em reômetro Brookfield DV - III, na taxa de cisalhamento de 30 rpm (similar à obtida nos processos de mastigação e deglutição do alimento), no tempo de leitura de 30s expressa em centipoise (cP) (Barros et al., 2008). A viscosidade em função do tempo foi obtida em intervalos de 5 minutos após o acréscimo do espessante industrializado, completando um tempo total de 20 minutos. A viscosidade de todas as preparações foi determinado à $25^{\circ} \mathrm{C}$, exceto o café, que foi à $75^{\circ} \mathrm{C}$. A medida da viscosidade em função da temperatura para a água e suco de laranja foi realizada a $5^{\circ} \mathrm{C}$ e $15^{\circ} \mathrm{C}$, para o café à $75^{\circ} \mathrm{C}$ e a $45^{\circ} \mathrm{C}$ para o leite integral e o leite espessado.

\subsection{Análise estatística}

Os resultados da solubilidade e poder de inchamento foram expressos na forma de média seguidos do desvio padrão. Os dados foram analisados pela ANOVA com o uso do software Statistical Analysis System (SAS) e as médias comparadas pelo teste de Tukey ao nível de 5\% de significância.

\section{Resultados e Discussão}

\subsection{Caracterização dos espessantes alimentares comerciais}

A partir das informações nutricionais dos espessantes alimentares comerciais e do amido de milho (Tabela 1) pôde-se observar alta porcentagem de carboidrato e sódio. Apenas o Nutilis ${ }^{\circledR}$ possui fibras alimentares na sua composição, com isso o valor calórico é menor. O valor energético atribuído aos espessantes se deve principalmente ao teor de carboidrato, uma vez que os demais constituintes são encontrados em baixas quantidades. 
Tabela 1. Informação nutricional dos espessantes alimentares comerciais e das preparações líquidas.

\begin{tabular}{|c|c|c|c|c|c|c|c|c|c|}
\hline \multirow[b]{2}{*}{$\begin{array}{l}\text { Informação } \\
\text { nutricional }\end{array}$} & \multicolumn{4}{|c|}{ Espessantes comerciais $(100 \mathrm{~g})$} & \multicolumn{5}{|c|}{ Preparações líquidas (100 mL) } \\
\hline & $\begin{array}{l}\text { Amido } \\
\text { de milho }\end{array}$ & $\begin{array}{c}\text { Resource } \\
\text { Thicken } \\
\text { Up® }\end{array}$ & Nutilis $®$ & $\begin{array}{c}\text { Thick \& } \\
\text { Easy® }\end{array}$ & Água & $\begin{array}{l}\text { Suco de } \\
\text { Laranja }\end{array}$ & $\begin{array}{c}\text { Leite } \\
\text { integral }\end{array}$ & $\begin{array}{c}\text { Leite } \\
\text { espessado }\end{array}$ & Café \\
\hline Valor energético (kcal) & 340 & 356 & 333 & 375 & 0 & 55 & 60 & 175,5 & 0 \\
\hline Carboidratos (g) & 85 & 89 & 83 & 100 & 0 & 12,25 & 5 & 24,5 & 0 \\
\hline Proteínas (g) & 0 & 0 & 0 & 0 & 0 & 0 & 3 & 10,28 & 0 \\
\hline Gorduras Totais $(\mathrm{g})$ & 0 & 0 & 0 & 0 & 0 & 0 & 3 & 4,13 & 0 \\
\hline Gorduras Saturadas (g) & 0 & 0 & 0 & 0 & 0 & 0 & 2 & 2,6 & 0 \\
\hline Gorduras Trans (g) & 0 & 0 & 0 & 0 & 0 & 0 & 0 & 0 & 0 \\
\hline Colesterol (mg) & 0 & 0 & 0 & 0 & 0 & 0 & 15 & 21,68 & 0 \\
\hline Fibra alimentar $(\mathrm{g})$ & 0 & 0 & 10 & 0 & 0 & 0 & 0 & 0 & 0 \\
\hline Cálcio (mg) & 0 & 0 & 0 & 0 & 0 & 0 & 126 & 381,75 & 0 \\
\hline Sódio (mg) & 0 & 222 & 153 & 125 & 0 & 3,6 & 60 & 148,5 & 0 \\
\hline
\end{tabular}

Fonte: Autores.

A Tabela 2 apresenta a quantidade de produto necessária para o espessamento, considerando as consistências recomendadas pelo NDD (National Dysphagia Diet Task Force, 2002). Observou-se que quanto maior a consistência (pudim > mel > néctar) que se deseja atingir, maiores quantidades de produto precisam ser adicionadas. Dentre os espessantes comerciais analisados, o Thick \& Easy® é o que necessita a adição de menores quantidades de produto em todas as consistências verificadas.

A adição de maior quantidade de produto pode afetar a aceitabilidade dos pacientes aos alimentos espessados, uma vez que podem deixar gosto residual, metálico, adstringente e/ou sabor amiláceo (Lotong et al., 2003). Matta et al. (2006) avaliando quatro espessantes alimentares comerciais adicionados em bebidas, demonstraram que os espessantes à base de amido conferiram uma textura granulada e um sabor de amido a todas as bebidas espessadas em consistências semelhantes a néctar e mel. Já os espessantes à base de goma não produziram textura granulada e o sabor do amido, quando presente, foi significativamente menor.

Tabela 2. Recomendação para espessamento de líquidos finos com espessantes alimentares comerciais.

\begin{tabular}{cccc}
\hline Consistência* & Resource Thicken Up® & Nutilis ${ }^{\circledR}$ & Thick \& Easy® \\
\hline Néctar & $5 \mathrm{~g}$ & $6 \mathrm{a} 9 \mathrm{~g}$ & $4,5 \mathrm{~g}$ \\
Mel & $10 \mathrm{~g}$ & $9 \mathrm{a} 12 \mathrm{~g}$ & $6,75 \mathrm{~g}$ \\
Pudim & $15 \mathrm{~g}$ & $12 \mathrm{a} 15 \mathrm{~g}$ & $9,0 \mathrm{~g}$ \\
\hline
\end{tabular}

*Níveis de consistências recomendados no "National Disphagia Diet" para tratamento da disfagia, sendo: Néctar de 51 a 350 cP; Mel de 351 a $1750 \mathrm{cP}$ e Pudim superior a $1750 \mathrm{cP}$.

Fonte: Autores.

\subsection{Microscopia}

A Figura 1 demonstra as imagens microscópicas obtidas para os espessantes alimentares comerciais e o amido de milho. A Figura 1A mostra a estrutura do amido de milho não modificado, íntegro e completamente corado de azul pelo lugol. Já nas Figuras 1B, 1C, pode-se verificar que o amido modificado, presente nos espessantes comerciais, corou-se de cor avermelhada e a estrutura do grão apresenta sinais de rompimentos e quebras.

O amido na forma nativa apresenta certas limitações que dificultam a sua aplicação. Contudo, a modificação dos amidos pode melhorar suas propriedades funcionais, conferindo maior poder de espessamento, gelificação, melhora na 
retenção de água e aumento da estabilidade, possibilitando amplo uso pela indústria alimentícia (BeMiller 1997; Silva et al., 2020).

Figura 1. Caracterização do amido de milho cru (A) e dos espessantes alimentares comerciais, Resource Thicken Up® (B), Nutilis® (C) e Thick \& Easy® (D) em microscopia ótica, corados com Lugol, aumento de 40X.

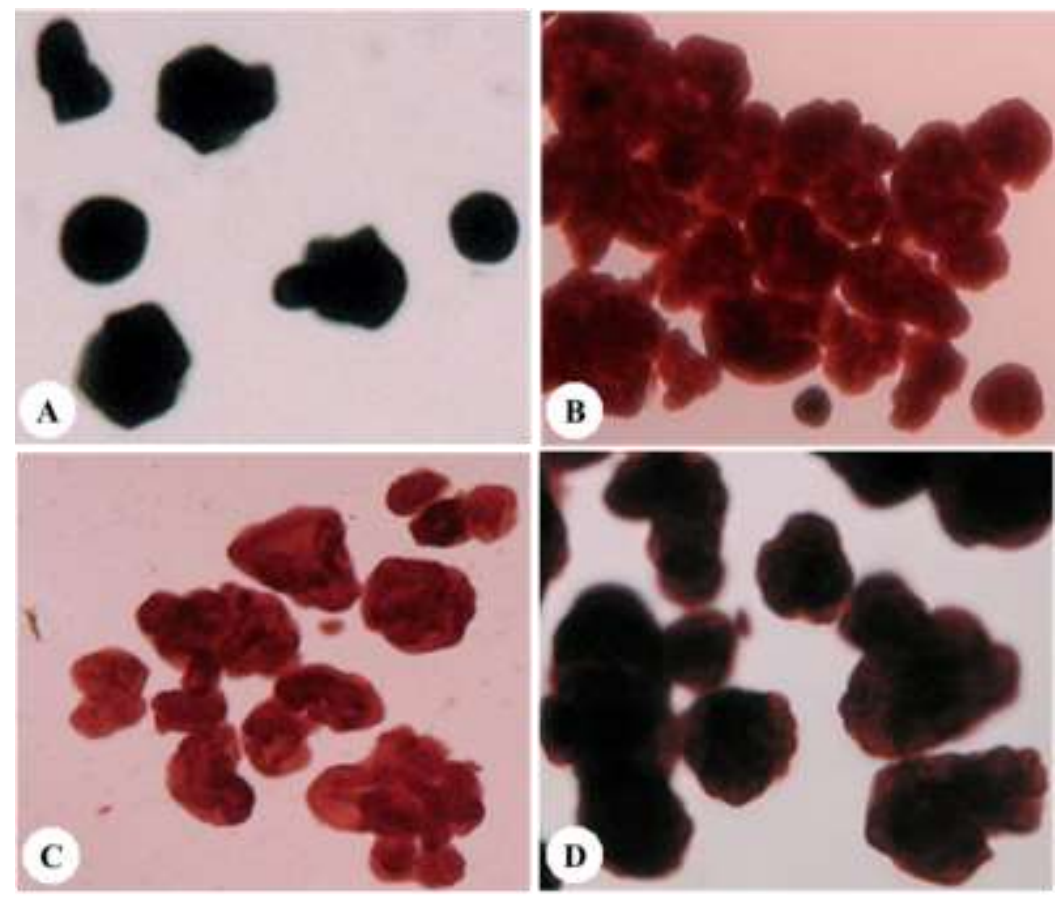

Fonte: Autores.

\section{$3.3 \mathrm{pH}$}

Na Tabela 3 pode-se verificar a variação de $\mathrm{pH}$ das preparações utilizadas para o espessamento, onde os maiores valores foram encontrados para a água, leite e leite espessado e os menores valores para o café e o suco de laranja. Os espessantes alimentares comerciais promoveram mudanças no $\mathrm{pH}$ das preparações, variando de neutro a ácido, sendo o suco de laranja mais ácido.

Tabela 3. Valores de $\mathrm{pH}$ dos alimentos espessados e valores destes adicionados a amido de milho e aos espessantes alimentares comerciais*

\begin{tabular}{|c|c|c|c|c|c|}
\hline \multirow[b]{2}{*}{ Preparações } & \multirow[b]{2}{*}{$\begin{array}{c}\mathrm{pH} / \\
\text { temperatura }\end{array}$} & \multirow[b]{2}{*}{ Amido de milho } & \multicolumn{3}{|c|}{ Espessantes alimentares comerciais } \\
\hline & & & $\begin{array}{c}\text { Resource } \\
\text { Thicken Up® }\end{array}$ & Nutilis® & $\begin{array}{c}\text { Thick \& } \\
\text { Easy® }\end{array}$ \\
\hline Água & 7,0 a $26^{\circ} \mathrm{C}$ & $5,60 \pm 0,05$ & $6,78 \pm 0,03$ & $6,08 \pm 0,03$ & $5,40 \pm 0,03$ \\
\hline Suco de laranja & 3,55 a $26,8^{\circ} \mathrm{C}$ & $4,06 \pm 0,01$ & $4,11 \pm 0,01$ & $4,15 \pm 0,03$ & $4,13 \pm 0,00$ \\
\hline Leite integral & 6,62 a $26,5^{\circ} \mathrm{C}$ & $6,56 \pm 0,01$ & $6,65 \pm 0,01$ & $6,63 \pm 0,01$ & $6,64 \pm 0,01$ \\
\hline Leite integral com Sustagem ${ }^{\circledR}$ & 6,42 a $25,5^{\circ} \mathrm{C}$ & $6,46 \pm 0,01$ & $6,51 \pm 0,00$ & $6,49 \pm 0,01$ & $6,49 \pm 0,00$ \\
\hline Café & 5,64 a $27,4{ }^{\circ} \mathrm{C}$ & $5,49 \pm 0,00$ & $5,64 \pm 0,01$ & $5,62 \pm 0,00$ & $5,59 \pm 0,00$ \\
\hline
\end{tabular}

Fonte: Autores.

\subsection{Poder de inchamento e solubilidade}

O poder de inchamento e a solubilidade dos grânulos para os espessantes e para o amido de milho cru estão apresentados na Tabela 4. O amido de milho cru tem baixo poder de inchamento e solubilidade em água, mas quando pré- 
gelatinizado a solubilidade em água e a formação de gel aumenta (Clerici, 2012), o que pode ser observado nos maiores valores de inchamento dos três espessantes comerciais, que contêm amido modificado em sua composição.

A solubilidade do amido está ligada a presença de amido danificado e moléculas de dextrinas ou maltodextrinas de baixo peso molecular, que são capazes de se tornarem solúveis em água (Barros et al., 2020), a presença destes ingredientes nas formulações mostram o aumento significativo da solubilidade em relação ao amido de milho cru, sendo que o Nutilis® foi o que apresentou maior solubilidade, diferindo em relação ao Resource Thicken Up® e Thick \& Easy®. Estes resultados podem indicar que os espessantes Resource Thicken Up® e Thick \& Easy® apresentam comportamento semelhante em relação ao poder de inchamento e solubilidade, já o Nutilis® apresenta maior solubilidade e menor poder de inchamento.

Tabela 4. Valores do poder de inchamento e solubilidade dos grânulos dos espessantes.

\begin{tabular}{lcccc}
\hline \multicolumn{1}{c}{ Parâmetros } & Amido de milho & Resource Thicken Up® & Nutilis ${ }^{\circledR}$ & Thick \& Easy® \\
\hline Poder de inchamento & $2,69^{\mathrm{c}} \pm 0,30$ & $20,39^{\mathrm{a}} \pm 0,98$ & $14,50^{\mathrm{b}} \pm 0,76$ & $20,26^{\mathrm{a}} \pm 1,04$ \\
Solubilidade dos grânulos & $0,42^{\mathrm{c}} \pm 0,09$ & $5,13^{\mathrm{b}} \pm 0,70$ & $33,89^{\mathrm{a}} \pm 1,04$ & $6,44^{\mathrm{b}} \pm 1,32$ \\
\hline
\end{tabular}

* Médias seguidas de letras diferentes, na horizontal, diferem significativamente entre si $(\mathrm{p}<0,05)$, pelo teste de Tukey. Fonte: Autores

\subsection{Viscosidade em função do tempo e temperatura}

A viscosidade das cinco preparações líquidas (água, suco de laranja, leite integral, leite espessado e café) foram aferidos, primeiramente, a $25^{\circ} \mathrm{C}$, com exceção do café que foi a $75^{\circ} \mathrm{C}$, nos tempos 5, 10, 15 e 20 minutos, nas preparações adicionadas de 1, 2, 3 e 4 colheres de chá dos espessantes alimentares comerciais (Tabela 5). Obtidos os valores, a água e o suco de laranja foram submetidos a $5^{\circ} \mathrm{C}$ e $15^{\circ} \mathrm{C}$, o café a $75^{\circ} \mathrm{C}$, o leite e o leite espessado a $45^{\circ} \mathrm{C}$, considerando a temperatura habitual de consumo (Figura 2).

Foi encontrado diferenças entre as viscosidades dos espessantes alimentares comerciais (Tabela 5). A diferença nos valores de viscosidade dos alimentos espessados pode ser devido a vários fatores, entre os quais está a provável hidratação contínua do agente espessante (amido modificado), que pode promover ainda mais o aumento da viscosidade dos alimentos (Adeleye; Rachal, 2007).

Como observado na Tabela 5, o Nutilis® apresentou os maiores valores de viscosidades em todas as preparações, além de uma ampla variação em relação ao tempo. Enquanto o Thick \& Easy® e Resource Thicken Up® tiveram valores de viscosidade semelhantes com pequenas variações em relação ao tempo, com exceção do leite integral e o leite espessado, na qual variações maiores foram observadas quando adicionados três ou quatro colheres de chá dos espessantes.

Os valores mais altos refletem produtos mais viscosos, enquanto os valores médios mais baixos refletem produtos menos viscosos. Moreno et al. (2006) comparando a viscosidade de cinco diferentes líquidos espessados em consistências semelhantes a néctar ou mel com uma variedade de produtos espessantes demonstrou que a viscosidade era altamente dependente do tipo de produto de espessamento e do tempo que foi permitido para engrossar. 
Tabela 5. Valores de viscosidade medidas em $\mathrm{cP}$ de três espessantes industrializados em diferentes preparações em relação ao tempo em minutos.

\begin{tabular}{|c|c|c|c|c|c|c|c|c|c|c|c|c|}
\hline \multirow{3}{*}{ Preparações } & \multicolumn{12}{|c|}{ Quantidade de espessante alimentar comercial (colher de chá)* } \\
\hline & \multicolumn{4}{|c|}{ Resource Thicken Up® } & \multicolumn{4}{|c|}{ Nutilis ${ }^{\circledR}$} & \multicolumn{4}{|c|}{ Tick \& Easy® } \\
\hline & 1 & 2 & 3 & 4 & 1 & 2 & 3 & 4 & 1 & 2 & 3 & 4 \\
\hline \multicolumn{13}{|l|}{ Água a $25^{\circ} \mathrm{C}$} \\
\hline $5 \mathrm{~min}$ & 4 & 22 & 170 & 1400 & 230 & 960 & 2000 & 10200 & 3 & 14 & 280 & 1400 \\
\hline $10 \mathrm{~min}$ & 4 & 24 & 200 & 1600 & 440 & 1160 & 2800 & 11000 & 3 & 14 & 280 & 1400 \\
\hline $15 \mathrm{~min}$ & 4 & 24 & 200 & 1800 & 480 & 1360 & 4000 & 12800 & 4 & 32 & 280 & 1400 \\
\hline $20 \min$ & 4 & 24 & 280 & 1800 & 680 & 1480 & 5200 & 13600 & 10 & 40 & 280 & 1400 \\
\hline \multicolumn{13}{|c|}{ Suco de laranja a $25^{\circ} \mathrm{C}$} \\
\hline $5 \mathrm{~min}$ & 4 & 34 & 250 & 2000 & 20 & 300 & 2200 & 8200 & 4 & 44 & 200 & 2000 \\
\hline $10 \mathrm{~min}$ & 8 & 34 & 400 & 2000 & 20 & 330 & 3400 & 9400 & 7 & 60 & 280 & 2200 \\
\hline $15 \min$ & 8 & 34 & 400 & 4800 & 30 & 430 & 4800 & 10000 & 8 & 80 & 320 & 2400 \\
\hline $20 \min$ & 8 & 40 & 400 & 4800 & 34 & 520 & 6200 & 14200 & 10 & 80 & 400 & 2400 \\
\hline \multicolumn{13}{|c|}{ Leite Integral a $25^{\circ} \mathrm{C}$} \\
\hline $5 \mathrm{~min}$ & 6 & 44 & 600 & 2400 & 14 & 74 & 500 & 1800 & 14 & 88 & 240 & 4800 \\
\hline $10 \mathrm{~min}$ & 8 & 54 & 700 & 2600 & 28 & 180 & 1400 & 4000 & 24 & 150 & 520 & 6000 \\
\hline $15 \mathrm{~min}$ & 8 & 54 & 700 & 3000 & 44 & 380 & 1800 & 7800 & 10 & 190 & 1000 & 6600 \\
\hline $20 \mathrm{~min}$ & 8 & 78 & 800 & 5000 & 66 & 580 & 3200 & 11400 & 10 & 170 & 1000 & 7200 \\
\hline \multicolumn{13}{|c|}{ Leite espessado a $25^{\circ} \mathrm{C}$} \\
\hline $5 \min$ & 14 & 44 & 120 & 550 & 28 & 88 & 400 & 1000 & 14 & 50 & 160 & 600 \\
\hline $10 \mathrm{~min}$ & 20 & 44 & 200 & 600 & 40 & 100 & 600 & 1200 & 14 & 70 & 400 & 1600 \\
\hline $15 \min$ & 20 & 50 & 320 & 1440 & 44 & 120 & 1800 & 3400 & 15 & 94 & 600 & 3000 \\
\hline $20 \mathrm{~min}$ & 20 & 64 & 480 & 2400 & 46 & 140 & 1800 & 3600 & 12 & 142 & 800 & 5000 \\
\hline \multicolumn{13}{|l|}{ Café a $75^{\circ} \mathrm{C}$} \\
\hline $5 \mathrm{~min}$ & 10 & 28 & 200 & 1400 & 4 & 600 & 3400 & 5600 & 3 & 14 & 280 & 1600 \\
\hline $10 \mathrm{~min}$ & 10 & 32 & 240 & 1400 & 6 & 1040 & 9200 & 15400 & 3 & 18 & 320 & 2000 \\
\hline $15 \mathrm{~min}$ & 14 & 34 & 280 & 2000 & 7 & 1080 & 10000 & 18000 & 4 & 18 & 400 & 2000 \\
\hline $20 \mathrm{~min}$ & 20 & 50 & 280 & 2000 & 7 & 1080 & 11400 & $>20000$ & 4 & 18 & 400 & 2600 \\
\hline
\end{tabular}

*colher de chá $=5$ gramas. Fonte: Autores.

As preparações foram submetidas a diferentes temperaturas (Figura 2), onde novamente o Nutilis® apresentou os maiores valores de viscosidade e variação em função da quantidade de espessante utilizada. Em água (Figura 2A) e suco de laranja (Figura 2B) o Thick \& Easy® e Resource Thicken Up® apresentaram valores de viscosidades aproximados. Em relação às variações de temperatura observou-se que valores mais baixos provocam aumento de viscosidade, enquanto valores mais altos de temperatura diminuem a viscosidade de uma preparação.

Para Sopade et al. (2008) as diferenças de viscosidades entre água, leite e suco para as mesmas amostras, ocorre devido aos diferentes constituintes de cada amostra, que tem forte impacto nos resultados de viscosidade. As interações podem ocorrer entre o agente espessante e alguns constituintes, como a presença de pectina e ácidos (suco de laranja) e íons (alta concentração no suco de laranja e leite). Além disso, a pectina presente no suco de laranja pode atuar como um agente espessante (Toneli et al., 2005) o que justifica a viscosidade de todas as amostras de espessantes com suco de laranja terem ficado mais viscosas do que em água. Alguns autores sugeriram que as viscosidades das bebidas espessadas à base de amido são diferentes daquelas das bebidas espessadas à base de goma, principalmente devido ao processo de espessamento diferente (Garcia et al., 2005; Sopade et al., 2008).

De forma geral, o Nutilis ${ }^{\circledR}$ apresentou maior poder de espessamento, e os resultados demonstram que o tempo de repouso após sua adição nas preparações é fundamental, uma vez que a viscosidade aumenta com o repouso. Enquanto o 
Resource Thicken Up® e o Thick \& Easy® apresentaram menores variações de viscosidade em relação ao tempo e às diferentes temperaturas. Pode-se concluir que temperaturas menores provocam um aumento de viscosidade, enquanto temperaturas maiores causam uma diminuição de viscosidade.

Figura 2. Viscosidade medida em centipoise $(\mathrm{cP})$ a diferentes temperaturas em água, suco de laranja, leite integral e leite espessado com a adição de uma a quatro colheres de chá (5 gramas cada) de três diferentes espessantes industrializados.

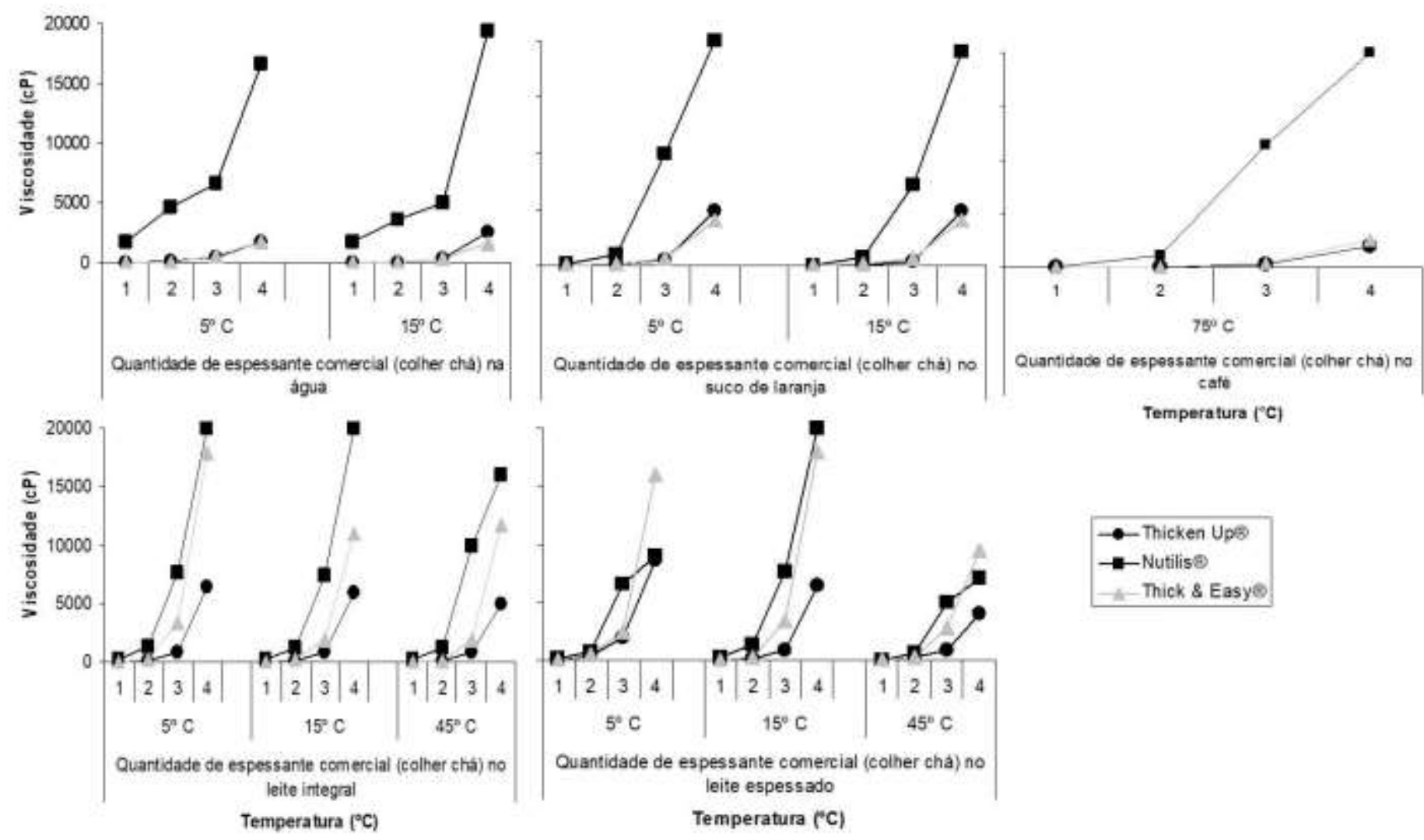

Fonte: Autores.

Atualmente há um vasto arsenal de espessantes industrializados que contribuem na consistência adequada para disfágicos, além de aumentar a densidade calórica dos alimentos e preparações, contribuindo para recuperação ou ganho de peso do paciente. A escolha do agente espessante é fundamental para a obtenção de consistências homogêneas e duráveis tentando alcançar máxima nutrição e qualidade dos mesmos, sendo que os espessantes devem interferir o menos possível nas propriedades organolépticas desses líquidos.

\section{Conclusão}

O espessamento dos alimentos líquidos é importante para o tratamento da disfagia orofaríngea, pois favorece o controle oral sobre os alimentos e promove melhoria do processo de deglutição. As preparações alimentares espessadas analisadas apresentaram viscosidades diferentes, dependendo da matriz alimentar, da temperatura, do tempo e da quantidade de produto adicionado. No entanto, mais trabalhos devem ser realizados com aplicação de análise sensorial dos alimentos espessadas com indivíduos disfágicos. 


\section{Agradecimentos}

Os autores agradecem à Coordenação de Aperfeiçoamento de Pessoal de Nível Superior (CAPES) pelo apoio financeiro (código 001), e ao Conselho Nacional de Desenvolvimento Científico e Tecnológico (CNPq) pela bolsa de doutorado de B. L. Tagliapietra (Processo 140805/2020-6).

\section{Referências}

Adeleye, B., \& Rachal, C. (2007). Comparison of the Rheological Properties of Ready-to-Serve and Powdered Instant Food-Thickened Beverages at Different Temperatures for Dysphagic Patients. Journal of the American Dietetic Association, 107(7), 1176-1182. https://doi.org/10.1016/j.jada.2007.04.011

Andersen, U. T., Beck, A. M., Kjaersgaard, A., Hansen, T., \& Poulsen, I. (2013). Systematic review and evidence based recommendations on texture modified foods and thickened fluids for adults ( $\geq 18$ years) with oropharyngeal dysphagia. E-SPEN Journal, 8(4), e127-e134. https://doi.org/10.1016/j.clnme.2013.05.003

Barros, S. L., Santos, N. C., Melo, M. O. P., Nascimento, A. P. S., Sousa, F. M., Santos, R. M. S. \& Figueirêdo, D. V. P. (2020). Qualidade físico-química e textural de geleias elaborados com morango, pimenta e maltodextrina. Research, Society and Development, 9(1), e153911868. http://dx.doi.org/10.33448/rsdv9i1.1868

Barros, S. P., Manzano, F. M. \& Silva, L. B. C. (2008) Manual de técnicas e receitas para espessamento de alimentos: utilização de diferentes amidos espessantes, Bauru: USP, 19p.

Bemiller, J. N. (1997). Starch modification: challenges and prospects. Starch/Stärke, 49(4), 127-131. https://doi.org/10.1002/star.19970490402

Cichero, J. A. (2013). Thickening agents used for dysphagia management: effect on bioavailability of water, medication and feelings of satiety. Nutrition Journal, 12(54). https://doi.org/10.1186/1475-2891-12-54

Clerici, M. T. P. S. (2012). Physical and/or Chemical Modifications of Starch by Thermoplastic Extrusion. Thermoplastic Elastomers. https://doi.org/10.5772/34318

Dobak, S. \& Kelly, D. (2020). Tough Pill to Swallow: Postextubation Dysphagia and Nutrition Impact in the Intensive Care Unit. Nutrition in Clinical Pratice, 36(1), 80-87. https://doi.org/10.1002/ncp.10602

Ellul, M. A., Benjamin, L., Singh, B., Lant, S., Michael, B. D., Easton, A., Kneen, R., Defres, S., Sejvar, J., \& Solomon, T. (2020). Neurological associations of COVID-19. The Lancet. Neurology, 19(9), 767-783. https://doi.org/10.1016/S1474-4422(20)30221-0

Ertekin, C. \& Aydogdu, I. (2003). Neurophysiology of swallowing. Clinical Neurophysiology, 114(12), 2226-2244. https://doi.org/10.1016/S13882457(03)00237-2

Fernández, L., Cabrera, N., Fernández, D., \& Olcese, L. (2020). Disfagia en tiempos de COVID-19 Dysphagia in COVID-19 times. Rev. Otorrinolaringol.Cir. Cabeza Cuello.80, 385-394.

Garcia, J. M., Chambers, E., Matta, Z. \& Clark, M (2005). Viscosity Measurements of Nectar- and Honey-thick Liquids: Product, Liquid, and Time Comparisons. Dysphagia, 20(4), 325-335. https://doi.org/10.1007/s00455-005-0034-9

Kim, C-Y. \& Yoo, B. (2018). Rheological characterization of thickened protein-based beverages under different food thickeners and setting times. Journal of Texture Studies, 49(3), 293-299. https://doi.org/10.1111/jtxs.12332

Lima, M. S. de, Sassi, F. C., Medeiros, G. C. de, Ritto, A. P., \& Andrade, C. R. F. de. (2020). Evolução funcional da deglutição em pacientes com COVID-19 internados em UTI. CoDAS, 32(4), e20200222. https://doi.org/10.1590/2317-1782/20192020222

Logemann, J. A. (2007). Swallowing disorders. Best Practice and Research in Clinical Gastroenterology, 21 (4), 563-573. https://doi.org/10.1016/j.bpg.2007.03.006

Lotong, V., Chun, S. S., Chambers IV, E., \& Garcia, J. M. (2003). Texture and flavor characteristics of beverages containing commercial thickening agents for dysphagia diets. Journal of Food Science, 68(4), 1537-1541. https://doi.org/10.1111/j.1365-2621.2003.tb09680.x

Matta, Z., Chambers IV, E., Garcia, J. M., \& Helverson, J. M. G. (2006). Sensory Characteristics of Beverages Prepared with Commercial Thickeners Used for Dysphagia Diets. Journal of the American Dietetic Association, 106(7), 1049-1054. https://doi.org/10.1016/j.jada.2006.04.022

Moreno, C., García, M. J. \& Martinez, C. (2006). Grupo de Estudio de la Alimentación del Mayor. Situation and adequacy analysis of dysphagia diets at a regional hospital. Nutrición Hospitalaria, 21(1), 26-31.

Moret-Tatay, A., Rodríguez-García, J., Martí-Bonmatí, E., Hernando, I., \& Hernández, M. J. (2015). Commercial thickeners used by patients with dysphagia: Rheological and structural behaviour in different food matrices. Food Hydrocolloids, 51, 318-326. https://doi.org/10.1016/j.foodhyd.2015.05.019

National Dysphagia Diet Task Force (2002). National Dysphagia Diet: Standardization for Optimal Care. Chicago: American Dietetic Association, 47p.

Pereira, A. S., Shitsuka, D. M., Perreira, F. J., Shitsuka, R. (2018). Metodologia da pesquisa cientifica. Santa Maria/RS. Ed. UAB/NTE/UFSM.

Phua, J., Weng, L., Ling, L., Egi, M., Lim, C. M., Divatia, J. V., Shrestha, B. R., Arabi, Y. M., Ng, J., Gomersall, C. D., Nishimura, M., Koh, Y., \& Du, B. 
Research, Society and Development, v. 10, n. 5, e50610515244, 2021

(CC BY 4.0) | ISSN 2525-3409 | DOI: http://dx.doi.org/10.33448/rsd-v10i5.15244

(2020). Intensive care management of coronavirus disease 2019 (COVID-19): challenges and recommendations. The Lancet Respiratory Medicine, 8(5), 506517. https://doi.org/10.1016/S2213-2600(20)30161-2

Schmidt, H. \& Oliveira, V. R. (2015). Avaliação reológica e sensorial de espessantes domésticos em diferentes líquidos como alternativa na disfagia. Brazilian Journal of Food Technology, 18(1). https://doi.org/10.1590/1981-6723.3714

Silva, G. D. O., Takizawa, F. F., Pedroso, R. A., Franco, C. M. L., Leonel, M., Sarmento, S. B. S., \& Demiate, I. M. (2006). Características físico-químicas de amidos modificados de grau alimentício comercializados no Brasil. Ciencia e Tecnologia de Alimentos, 26(1), 188-197. https://doi.org/10.1590/S010120612006000100030

Silva, J. M., Junior, B. D., Vieira, V. B., Klososki, S. J., Barão, C. E., Pinheiro, K. H. \& Pimentel, T. C. (2020). Amido de mandioca modificado por oxidação: propriedades físicas e químicas e perfil de textura de géis. Research, Society and Development, 9(10), e9089108238. http://dx.doi.org/10.33448/rsdv9i10.8238

Sopade, P. A., Halley, P. J., Cichero, J. A. Y., Ward, L. C., Liu, J., \& Varliveli, S. (2008). Rheological characterization of food thickeners marketed in Australia in various media for the management of dysphagia. III. Fruit juice as a dispersing medium. Journal of Food Engineering, 86(4), 604-615. https://doi.org/10.1016/j.jfoodeng.2007.11.013

Sukkar, S. G., Maggi, N., Cupillo, B. T. \& Ruggiero, C. (2018). Optimizing Texture Modified Foods for Oro-pharyngeal Dysphagia: A Difficult but Possible Target? Frontiers in Nutrition, 5, 68. https://doi.org/10.3389/fnut.2018.00068

Toneli, J. T. C. L., Murr, F. E. X. \& Park, K. J. (2005). Review: estudo da reologia de polissacarídeos utilizados na indústria de alimentos. Revista Brasileira de Produtos Agroindustriais, 7(2), 181-204.

Wei, Y., Guo, Y., Li, R., Ma, A., \& Zhang, H. (2021). Rheological characterization of polysaccharide thickeners oriented for dysphagia management: Carboxymethylated curdlan, konjac glucomannan and their mixtures compared to xanthan gum. Food Hydrocolloids, 110, 106198. https://doi.org/10.1016/j.foodhyd.2020.106198 